ISSN: 2215-2644

revedu@gmail.com

Universidad de Costa Rica

Costa Rica

\title{
Estudio descriptivo de variables sociodemográficas y motivacionales asociadas a la deserción: la perspectiva de personas universitarias de primer ingreso
}

Hernández-Jiménez, María Teresa; Moreira-Mora, Tania Elena; Solís-Salazar, Martín; Fernández-Martín, Tatiana

Estudio descriptivo de variables sociodemográficas y motivacionales asociadas a la deserción: la perspectiva de personas universitarias de primer ingreso

Revista Educación, vol. 44, núm. 1, 2020

Universidad de Costa Rica, Costa Rica

Disponible en: http://www.redalyc.org/articulo.oa?id=44060092023

DOI: https://doi.org/10.15517/revedu.v44i1.37247

Esta obra está bajo una Licencia Creative Commons Atribución-NoComercial-SinDerivar 3.0 Internacional. 


\title{
Estudio descriptivo de variables sociodemográficas y motivacionales asociadas a la deserción: la perspectiva de personas universitarias de primer ingreso
}

\begin{abstract}
A Descriptive Study of Sociodemographic and Motivational Variables Associated with Dropping out of School: A Perspective of Freshman University Students
\end{abstract}

Maria Teresa Hernández-Jiménez

Instituto Tecnológico de Costa Rica, Costa Rica

thernandez@itcr.ac.cr

(iD) http://orcid.org/0000-0002-5672-5743

Tania Elena Moreira-Mora

Instituto Tecnológico de Costa Rica, Costa Rica

tmoreira@itcr.ac.cr

(iD) http://orcid.org/0000-0002-8955-0804

Martin Solis-Salazar

Instituto Tecnológico de Costa Rica, Costa Rica

marsolis@itcr.ac.cr

iD http://orcid.org/0000-0003-4750-1198

\section{Tatiana Fernández-Martín}

Instituto Tecnológico de Costa Rica, Costa Rica

tafema@itcr.ac.cr

iD http://orcid.org/0000-0002-7726-0068

\author{
DOI: https://doi.org/10.15517/revedu.v44i1.37247 \\ Redalyc: http://www.redalyc.org/articulo.oa?id=44060092023
}

Recepción: 31 Mayo 2019

Aprobación: 10 Noviembre 2019

\section{RESUMEN:}

El análisis del fenómeno de la deserción estudiantil en contextos universitarios resulta complejo por la interacción de múltiples factores sociales, familiares, individuales e institucionales. El propósito del estudio fue describir algunas variables sociodemográficas y motivacionales asociadas a la deserción de estudiantes de primer ingreso de programas de grado en las cohortes del 2011, 2012 y 2013, con estudiantes activos y desertores de una institución de educación superior estatal de Costa Rica. Para tal fin, se construyó un instrumento de preguntas cerradas y abiertas, el cual se validó con el juicio de expertos y un grupo focal. Posteriormente, con la técnica de la encuesta se aplicó el instrumento por vía WEB y telefónica a una muestra no probabilística de 519 estudiantes. Entre los resultados de la prueba chi-cuadrada se encontró que la condición de abandono se asoció con: no matricular la carrera que más deseaba, el poco interés por estudiar en la carrera en la cual fue admitido y el deseo de estudiar en otra institución. El principal hallazgo del estudio es que las variables motivacionales son las que más inciden en el abandono. A partir de estos resultados se recomienda una apropiada inducción vocacional, para reducir las probabilidades de deserción, así como generar una estrategia para mantener la motivación durante los dos primeros años de ingreso a la universidad.

Palabras clave: Deserción, Educación Superior, Variables Sociodemográficas, Motivación.

\section{Abstract:}

Analyzing the college dropout phenomenon is complex, given the different social, family, individual and institutional factors that come into play. This study attempts to uncover various sociodemographic and motivational variables associated with the reasons associated with freshman students who dropped out a college. The study is based on a cohort of from 2011,2012 and 2013, which included both active and those who had dropped out of a State Costa Rican University. A questionnaire was drafted that included both open and close-ended questions. The questionnaire was validated by a group of experts and a focal group. Afterwards, a nonprobabilistic sample of 519 students completed an online and phone survey. The results of a chi-square test reveal that dropping 
out of school was associated with not being able to enroll in a student's first choice major, lack of interest in the career path the student was admitted to and student desire to attend another college. The main finding in this study is that motivational values have the highest impact on a student's decision to withdraw from school. Based on the results, we recommend appropriate vocational testing to reduce student probability of dropping out and create a strategy to keep students motivated during their first two years of college.

KEYWORDS: Dropout, Higher education, Sociodemographic Characteristics, Motivation.

\section{INTRODUCCIÓN}

En el ámbito de la educación superior estatal una tarea obligatoria es el análisis del fenómeno de la deserción no solo para determinar las causas específicas que llevan al estudiantado a abandonar sus estudios, sino también determinar en qué medida la institución puede tomar acciones preventivas para reducir la tasa de abandono, sobre todo en estudiantes que inician sus estudios. En consecuencia, el propósito del estudio fue describir algunas variables sociodemográficas y motivacionales asociadas a la deserción de estudiantes de primer ingreso de programas de grado en las cohortes del 2011, 2012 y 2013 de esta universidad, con la finalidad de que las autoridades universitarias tomen medidas preventivas.

En estudios previos en la temática se ha encontrado que la falta de orientación vocacional en el estudiantado juega un papel fundamental, pues al no tener claridad en este aspecto a la hora en que las personas jóvenes inician sus estudios universitarios, desertan precozmente (Torres, 2018); así como si los beneficios otorgados por la universidad son percibidos por parte del estudiantado como superiores a los costos personales en términos de esfuerzo y dedicación, la decisión será permanecer en la institución, caso contrario desertará. Lo anterior implica una interacción directa entre el sistema académico y social de las universidades (Rodríguez y Zamora, 2014).

A pesar de que esta temática no es novedosa o ajena para quienes se encuentran inmersos en el entorno universitario, las tasas de deserción y bajas tasas de graduación se han convertido en un asunto de creciente interés para las instituciones de educación superior y las autoridades educativas en los últimos cincuenta años, dado que este fenómeno amplía la brecha social y económica, así como limita el desarrollo de la nación. Según Rodríguez y Zamora (2014) la deserción origina problemas sociales, institucionales y personales; desde el punto de vista social incide negativamente en los índices de pobreza, aumenta el desempleo, disminuye el aporte intelectual e incrementa el costo de la educación debido a la sub-optimización de los recursos. En relación con lo institucional afecta el cumplimiento de la misión de la organización, baja los índices de eficiencia, calidad e ingreso. En lo personal se percibe la sensación del fracaso, pérdida de oportunidades laborales implicando menor posibilidad de conseguir empleos satisfactorios y el impacto que esto produce en términos individuales y familiares. Es importante señalar que la cifra del estudiantado que deserta de sus estudios superiores a nivel latinoamericano ronda en promedio el 50\% (Torres, 2018). De ahí que uno de los desafíos de las instituciones de educación superior es crear los mecanismos para retener a la población estudiantil que ingresa al sistema universitario y evitar que deserte (Casanova, García y Miranda, 2018). Además, los tiempos de graduación y deserción estudiantil forman parte de discusión y análisis en los procesos de autoevaluación y acreditación de los programas académicos (Regueyra, 2013).

En el caso de la universidad estatal de Costa Rica, objeto de la presente investigación, se evidencia esta problemática en la Tabla 1, donde se compara el total de quienes ingresaron (MAT) con los no matriculados (NO MAT) en el segundo semestre para los años comprendidos entre 2010 y 2014. 
TABLA 1

Porcentaje de estudiantes de primer ingreso por año, que no matricularon en el segundo semestre del mismo año.

\begin{tabular}{|c|c|c|c|c|c|c|c|c|c|c|c|c|c|c|c|}
\hline \multirow{3}{*}{$\begin{array}{l}\text { Grado } \\
\text { académico }\end{array}$} & \multicolumn{15}{|l|}{ Año } \\
\hline & \multicolumn{3}{|l|}{2010} & \multicolumn{3}{|l|}{2011} & \multicolumn{3}{|l|}{2012} & \multicolumn{3}{|l|}{2013} & \multicolumn{3}{|l|}{2014} \\
\hline & MAT. & $\begin{array}{l}\text { NO } \\
\text { MAT. }\end{array}$ & $\%$ & MAT. & $\begin{array}{l}\text { NO } \\
\text { MAT }\end{array}$ & $\%$ & MAT. & $\begin{array}{l}\text { NO } \\
\text { MAT }\end{array}$ & $\%$ & MAT. & $\begin{array}{l}\text { NO } \\
\text { MAT. }\end{array}$ & $\%$ & MAT. & $\begin{array}{l}\text { NO } \\
\text { MAT. }\end{array}$ & $\%$ \\
\hline $\begin{array}{l}\text { Bachillerato } \\
\text { Universitario }\end{array}$ & 579 & 69 & $11,92 \%$ & 435 & 80 & $18,39 \%$ & 453 & 70 & $15,45 \%$ & 722 & 107 & $14,82 \%$ & 641 & 117 & $18,25 \%$ \\
\hline Licenciatura & 809 & 96 & $11,87 \%$ & 651 & 116 & $17,82 \%$ & 848 & 120 & $14,15 \%$ & 1.183 & 113 & $9,55 \%$ & 1.049 & 137 & $13,06 \%$ \\
\hline TOTAL & 1.388 & 165 & $11,89 \%$ & 1.086 & 196 & $18,05 \%$ & 1.301 & 190 & $14,60 \%$ & 1.905 & 220 & $11,55 \%$ & 1.690 & 254 & $15,03 \%$ \\
\hline
\end{tabular}

Fuente: Elaboración propia con datos proporcionados por el Departamento de Admisión y Registro.

Como lo muestran estos datos, el porcentaje de estudiantes de primer ingreso en carreras de grado que no se matricularon en el segundo semestre, osciló entre el 11,55\% y 18,05\% durante este periodo. Ante este contexto surgió el siguiente problema de la investigación: cómo se puede reducir el abandono de la población estudiantil de primer ingreso en esta universidad estatal considerando sus características sociodemográficas y motivacionales.

$\mathrm{Al}$ respecto, se han desarrollado múltiples investigaciones descriptivas y correlacionales acerca de las variables asociadas al fenómeno de la deserción, tales como los estudios de Aranzazu y Rojas (2018), Casanova et al. (2018), Rodríguez y Zamora (2014), Regueyra y Rojas (2013) y Treviño, Ibarra, Castán, Laria y Guzmán (2013), quienes coinciden en que la deserción estudiantil debe ser explicada a partir del análisis de múltiples factores: personales, familiares, económicos, institucionales y los referidos a los temas vocacionales.

\section{ANTECEDENTES}

En esta línea de investigación los estudios más recientes han analizado la deserción en el estudiantado de primer ingreso con la finalidad de predecir con precisión el riesgo de abandono universitario durante los primeros ciclos lectivos (Aranzazu y Rojas, 2018; Casanova et al. 2018). La deserción en el primer año, es uno de los indicadores más valorados a nivel internacional para evaluar la capacidad y la eficacia de las instituciones terciarias para retener a los jóvenes en el sistema educativo, considerando que la mayor deserción de estudiantes se suscita tempranamente (Casanova et al. 2018). Por su parte, Aranzazu y Rojas (2018), afirman que el mayor porcentaje de abandono se presenta en el primer año de estudio, las edades promedio de abandono oscilan entre 18 y 19 años, periodo en el cual la población estudiantil se encuentra en la llamada adolescencia tardía. Según los autores es en esta etapa de cambios donde se empieza a instaurar la autonomía tanto familiar como intelectual, lo cual conlleva en ocasiones a la toma errada de decisiones y por ende al descuido e indecisión académica.

Tales estudios se han ejecutado analizando el fenómeno en diferentes periodos, desde solo el primer año universitario hasta 5 años y más (Mesarić y Šebalj, 2016; Miranda y Guzmán, 2017), como en el caso de la Universidad Nacional, en donde se determinó un máximo de 18 ciclos lectivos, o sea 9 años como periodo de observación para que ocurra la deserción (Rodríguez y Zamora, 2014). Igualmente, los criterios para definir la deserción han sido variantes, desde dejar de matricular en el primer o segundo ciclo lectivo del primer año (Rodríguez y Zamora, 2014; Plagge, 2013; Aguiar, Ambrose, Chawla, Goodrich y Brockman, 2014 y Oñate, 2016). En esta investigación se asumió el criterio de la Comisión Nacional de Rectores de Costa Rica (CONARE), de contar con dos años consecutivos de no matrícula en la universidad para ser clasificado como estudiante desertor, ello según el esfuerzo conjunto entre la Cátedra UNESCO y el CONARE, desde el año 2008 . 
Además, ha sido de interés en este tipo de investigaciones la inclusión de diversas variables que se han asociado a la deserción, tales como: aquellas relacionadas con las características académicas, institucionales, financieras y sociodemográficas (Delen, 2010 y Torres, 2018), puntuación obtenida en las pruebas de ingreso a la universidad (Oñate, 2016), lugar de residencia (Jia, 2013; Nakhkob y Khademi, 2016), colegio de procedencia (Oztekin, 2016), planes académicos (Jia, 2013). El conjunto de estos hallazgos ha proporcionado información importante acerca de las variables individuales e institucionales que incrementan la probabilidad de deserción y que esta es el resultado de procesos complejos, donde las responsabilidades radican tanto en el estudiantado como en las propias instituciones y que afectan a ambos grupos de actores. (Casanova et al., 2018).

En un estudio elaborado en la Universidad Nacional de Colombia, similar al contexto de la universidad objeto del presente estudio, tanto en cifras de estudiantes matriculados como en sus programas académicos en ingeniería, se identificó que la mayoría del estudiantado se incorpora inmediatamente después de culminados sus estudios de educación media, por lo que se encuentra en una edad donde se tiene mayor vulnerabilidad y susceptibilidad al cambio y a la transición, por lo cual es necesario que la universidad implemente estrategias enfocadas en el primer año de estudio, dirigidas a la permanencia y el acompañamiento estudiantil (Aranzazu y Rojas, 2018).

En el contexto de la universidad estatal de Costa Rica de esta investigación, la deserción estudiantil ha sido analizada desde los primeros años de la institución. La Dirección de Servicios Estudiantiles y Académicos (DISEA, 1980a, 1980b y 1981) identificó como causas de deserción esencialmente las referidas a insatisfacción con la carrera, traslados a otras universidades, principalmente a la Universidad de Costa Rica y problemas económicos. En estudios posteriores Barquero (1993 y 1997) efectúa una sistematización de las principales causas de abandono, a saber: problemas laborales (49\%), problemas económicos (22\%), problemas familiares (16\%) y traslado a otra universidad (13\%), entre otros. Un elemento importante identificado en estos estudios es que alrededor del $50 \%$ del estudiantado que se retiraba de la institución lo hacía en los primeros dos años de carrera.

\section{MARCo TEÓRICO}

Una precisión conceptual y operativa del abandono escolar resulta compleja, por no existir un único concepto de este fenómeno que dimensione toda su complejidad y por la multiplicidad de formas de abandono de los estudios en la educación superior estatal. Como lo destacan Regueyra y Rojas (2013), no existe un único modelo de referencia para abordar el fenómeno de la deserción, por lo que el modelo de estudio que se elija dependerá de las condiciones institucionales y del contexto; sin embargo, se debe rescatar que las autoras se refieren al estudio de cohortes como un método acertado para el análisis. Las dificultades de conceptualización al respecto fueron también señaladas previamente por Tinto (1989), quien afirmó que el estudio de la deserción de la educación superior es extremadamente complejo, pues implica no solo una variedad de perspectivas, sino también una gama de diferentes tipos de abandono. Adicionalmente, afirma que ninguna definición puede captar en su totalidad la complejidad de este fenómeno, quedando en manos de los investigadores la elección de la definición que mejor se ajuste a sus objetivos y al problema a investigar.

En el caso de Costa Rica, Brenes (2005) desarrolla un estudio para el Consejo Nacional de Rectores [CONARE], en el cual se distinguen los siguientes tipos de deserción:

- Deserción institucional: abandono de los estudios en una determinada institución.

- Deserción del sistema universitario estatal: abandono de los estudios en el sistema universitario estatal.

- Deserción del sistema de educación superior: abandono de todo el sistema de educación superior.

- Deserción de carrera: abandono de la carrera en la que estaba empadronado el o la estudiante. 
- Deserción real: número de estudiantes que ingresan en el año t a una determinada carrera, universidad o sistema de educación superior, menos las y los estudiantes de esta cohorte que ya se graduaron en esa carrera, universidad o sistema de educación superior, y los que aún permanecen estudiando.

- Deserción específica ajustada: deserción que se corrige asumiendo que la mitad del estudiantado que aún permanece estudiando finalmente termina su carrera.

Por su parte, en un estudio desarrollado en la Universidad Nacional de Costa Rica, define dos tipos de estudiantes desertores, las y los tempranos y las y los itinerantes. Los primeros son aquellos que matriculan en el primer ciclo y/o segundo ciclo del año de ingreso y no han vuelto a matricular hasta la fecha de corte del estudio y los segundos son estudiantes que han presentado una matrícula irregular y además presentan inactividad académica en el último año (Rodríguez y Zamora, 2014).

Considerando estos planteamientos teóricos y la dificultad implícita en la medición de la deserción total de una institución de educación superior, en esta investigación se entenderá por abandono o interrupción de los estudios universitarios cuando un estudiante no matricula por un período de dos años consecutivos, a partir de la matrícula inicial en la institución. Esta definición se fundamenta en un acuerdo del Consejo Nacional de Rectores (2008), que establece que un desertor es aquel que no matricula ningún curso en la universidad por un periodo consecutivo de dos años. Con base en esta definición se clasificaron a los participantes del estudio en:

- No desertor: incluye estudiantes que se mantienen activos en la institución o lograron concluir sus estudios.

- Desertor primer ingreso: incluye estudiantes que matricularon su primer semestre en la institución y no matricularon semestres posteriores, alcanzando al inicio del 2016 un mínimo de dos años consecutivos sin matricular.

Por otra parte, González (2006) destaca que la deserción se ha analizado desde tres perspectivas. Desde una óptica interna referida a los procesos de enseñanza (calidad educativa, estructura y pertinencia curricular, formación docente y otros). La segunda con un énfasis en los procesos externos a lo propiamente educativo (características del estudiantado, situación socioeconómica del país, acceso a financiamiento y otros). Mientras que la tercera, centrada en la interacción entre ambos tipos de factores. Específicamente, en esta investigación se asumió la segunda perspectiva, analizando las siguientes características del estudiantado asociadas a la deserción:

Sociodemográficas: Esta categoría incluyó variables indicativas de la condición económica, la tenencia de hijos, nivel educativo del núcleo familiar, la condición de becados en el colegio, el apoyo financiero o moral de otras personas y condición laboral del estudiantado.

Motivacional: En este estudio se midió, específicamente, el interés por continuar sus estudios en la universidad y en la carrera seleccionada. Tales variables que incidirían en la motivación del alumnado serían: el sentido que el alumnado asigna a las tareas académicas, la relevancia de los estudios en relación con sus metas futuras y el desafío académico de la carrera, entre otros aspectos (Canales y De los Ríos, 2007).

\section{Metodología}

El estudio se elaboró desde un enfoque metodológico cuantitativo basado en un diseño descriptivo para identificar algunas variables sociodemográficas y motivacionales asociadas a la decisión de abandonar o continuar sus estudios en tres cohortes de la universidad. 


\section{Muestra}

Los criterios de selección de estas tres cohortes 2011, 2012 y 2013 fueron dos: analizar este fenómeno en poblaciones recientes, bajo el supuesto de que las características de esta población pueden ser similares con la población futura de esta universidad y, considerar el criterio de dos años consecutivos de no matrícula en la universidad. Específicamente, de esta población de primer ingreso se identificó:

- Sexo: el $65 \%$ de la población es hombre y el 35\% es mujer.

- Edad, según rango: el 92\% se concentra en el rango de edad de 18 a 22 años, el 4\% de 23 a 26 años, el $2 \%$ menos de 18 años y el $2 \%$ más de 26 años.

- Nacionalidad: el $96,81 \%$ es costarricense y el porcentaje restante proceden de 20 países diferentes: Nicaragua, Colombia, México, Venezuela, Guatemala, Perú, China, Alemania, Canadá, Corea, Bolivia, República Checa, España, Estados Unidos, Francia, Honduras, Panamá, El Salvador y Taiwán.

- Lugar de residencia, según provincia de procedencia: el 32\% de San José, el 29\% de Cartago, el 20\% de Alajuela, el 8\% de Heredia, el 4\% de Guanacaste, el 4\% de Puntarenas y el 3\% de Limón.

- Índice de Desarrollo Social (IDS), según distrito de procedencia: el 54\% del estudiantado procedía del nivel de mayor desarrollo (72,5-100), un 33\% de nivel medio (58-72,4), un $11 \%$ del nivel bajo $(43,9-57,9)$ y un $2 \%$ del nivel muy Bajo $(0-43,8)$.

El total de las tres cohortes fue de 4989 estudiantes de primer ingreso, a quienes se les envió el instrumento; el cual fue contestado por una muestra no probabilística de 129 desertores vía WEB y telefónica, mientras que solo vía WEB un total de 390 estudiantes activos. El dato específico de los encuestados no se determinó por ser anónima, quienes aceptaron participar de manera voluntaria. Esta aplicación se ejecutó entre setiembre y octubre del 2016 mediante la técnica de la encuesta.

\section{INSTRUMENTO}

Con la finalidad de recopilar datos sobre las variables de interés en el contexto específico de esta universidad, se construyó un cuestionario que se validó con la técnica de jueces y un grupo focal para obtener evidencias asociadas al contenido, específicamente, para determinar el grado de representatividad y pertinencia de las variables que se deseaban medir. Los expertos efectuaron observaciones relacionadas con el formato, la organización de los ítems y su redacción. Luego se aplicó el instrumento a un grupo focal de estudiantes para verificar el nivel de comprensión de las preguntas y probar el sistema de LimeSurvey. La versión final del cuestionario quedó conformada por un total de 33 preguntas con diferentes opciones de respuesta, las cuales se organizaron por categorías, como se describe a continuación:

- Preguntas cerradas de la 1 a la 13: carrera seleccionada, motivos para continuar en la carrera y en la universidad y razones relacionadas con el interés por sus estudios.

- Preguntas cerradas de la 14 a la 30: condición laboral, condición económica, la tenencia de hijos, nivel educativo del núcleo familiar, la condición de becados en el colegio, el apoyo financiero o moral de otras personas, tiempo de traslado a la universidad, lugar de residencia y acompañamiento por pares.

- Preguntas abiertas 31 a 33: razones de la disminución del interés de estudiar en la universidad y en la carrera seleccionada y de la selección de una carrera que no era su opción vocacional.

Los participantes seleccionaban la opción que mejor describía sus propias vivencias y percepciones de la universidad y la carrera a la que ingresó. 


\section{Resultados}

En este estudio se presentan los datos descriptivos de las variables medidas en la encuesta aplicada a estudiantes de primer ingreso de programas de grado en las cohortes del 2011, 2012 y 2013, tanto a estudiantes activos como desertores. En la Tabla 2 se indica aquellas que presentaron una asociación con la condición de abandono a un nivel de significancia de $\mathrm{p}<0.05\left(^{*}\right)$ de la prueba de chi-cuadrada. 
María Teresa Hernández-Jiménez, et al. Estudio descriptivo de VARiables sociodemográficas y motiva...

TABLA 2

Porcentajes de estudiantes desertores y no desertores de las cohortes 2011, 2012 y 2013 según interés en estudiar en la carrera y en la universidad (U).

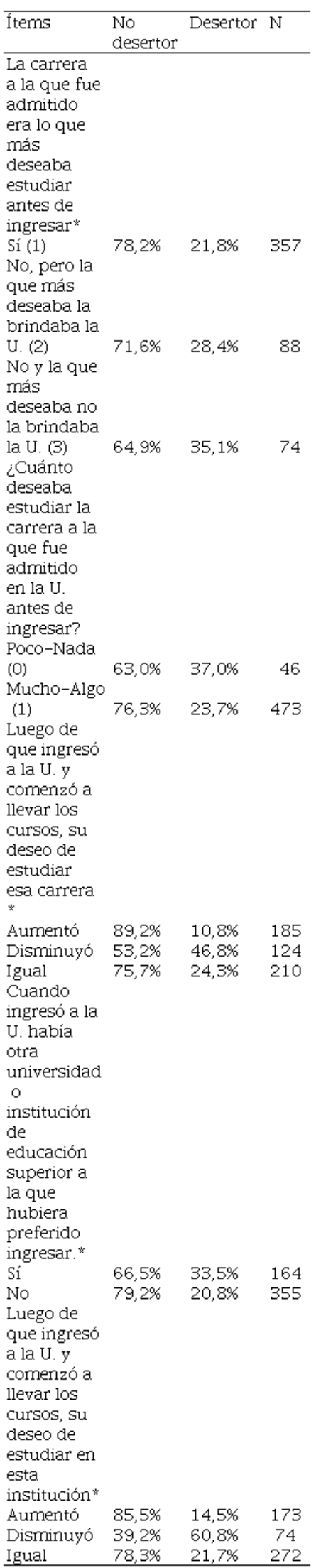


Fuente: Elaboración propia.

En general, de la muestra de las tres cohortes que ingresó a la universidad en estudio, se observa en la Tabla 2 que el 35,1\% de quienes no matricularon la carrera que más deseaba y no era parte de la oferta académica de esta universidad abandonó sus estudios. También desertaron en un 37\% quienes mostraron poco o nada de interés por estudiar en la carrera en que fue admitido y un $46,8 \%$ aquellos cuyo desinterés aumentó. Por otra parte, el 33,5\% de quienes manifestaron el deseo de estudiar en otra institución de educación superior y el 60,8\% de los que indicaron que su interés de estudiar disminuyó, también desertaron. De estas variables, cuatro se asociaron con la condición de deserción. Además, de los 164 estudiantes que no tenían como principal objetivo ingresar a esta universidad estatal, el 87\% manifestó su interés de estudiar en la Universidad de Costa Rica.

TABLA 3

Razón por la cual le disminuyó el interés de estudiar en la universidad (U) luego de que ingresó (n=74)

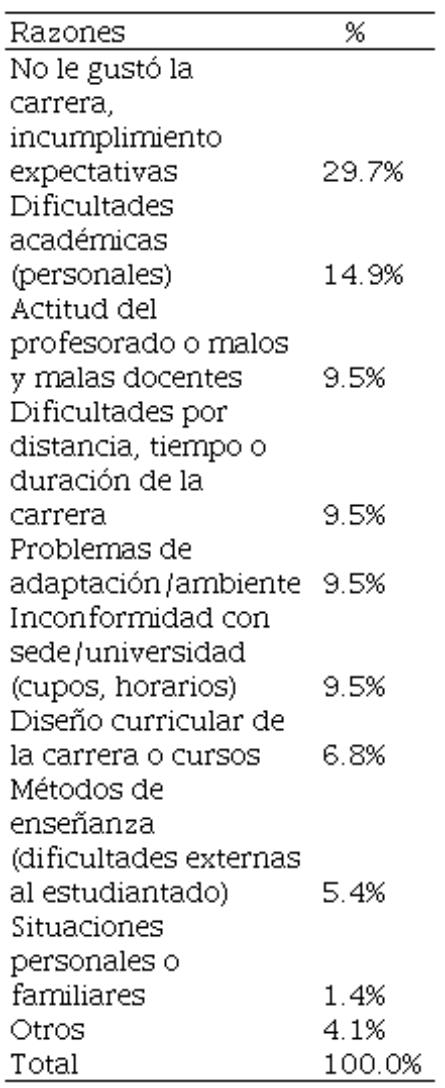

Fuente: Elaboración propia.

En esta Tabla 3 se resumen las respuestas abiertas de los 74 estudiantes que reportaron una disminución de su deseo de estudiar en la institución. Una tercera parte señaló que no se cumplieron sus expectativas y un 25,8\% apuntó a aspectos más específicos de la oferta académica de la carrera, tales como: cupos, horarios, diseño curricular y duración. En este punto algunos y algunas manifestaron:

E32 (comunicación personal, 17 de setiembre, 2016): Cuando estaba en el colegio no estaba seguro que quería estudiar. Entré y en los primeros cursos de carrera me di cuenta que la opción que escogí no era lo mío.

E430 (comunicación personal, 9 de setiembre, 2016): Porque el nivel de estudio era muy difícil, los créditos son muy pesados, y todas las pruebas se realizaban en las mismas fechas, como exámenes y proyectos. 
$\mathrm{Al}$ igual que en la pregunta anterior, en la Tabla 4 se puede reconocer que la principal razón de la pérdida de interés por continuar en la carrera para casi la mitad de los desertores $(46,6 \%)$ fue que no le gustaba y no llenaba sus expectativas. Mientras que para un $20 \%$ las razones estaban más relacionadas con las y los docentes (actitudes y método de enseñanza). Como lo destacó un participante:

E126 (comunicación personal, 8 de setiembre, 2016): El ambiente en general. La mayoría de profesores también me disminuyeron el deseo de seguir estudiando esa carrera, por su forma de enseñar en algunas ocasiones un poco drástica. No sentía la pasión que veía en algunos de mis compañeros y conocidos. No me sentía pleno.

\section{TABLA 4}

Razón por la cual le disminuyó el interés de estudiar la carrera luego de que ingresó $(\mathrm{n}=124)$

\begin{tabular}{lc}
\hline Razones & $\%$ \\
\hline $\begin{array}{l}\text { No le gustó la } \\
\text { carrera, } \\
\text { incumplimiento } \\
\text { expectativas }\end{array}$ & $46.6 \%$ \\
$\begin{array}{l}\text { Dificultades } \\
\text { académicas } \\
\text { (personales) }\end{array}$ & $13.8 \%$ \\
$\begin{array}{l}\text { Actitud profesorado } \\
\text { o malos y malas } \\
\text { docentes }\end{array}$ & $10.3 \%$ \\
$\begin{array}{l}\text { Métodos de } \\
\text { enseñanza } \\
\text { (dificultades externas }\end{array}$ & \\
$\begin{array}{l}\text { al estudiantado) } \\
\text { Diseño curricular de } \\
\text { la carrera o cur'sos }\end{array}$ & $9.5 \%$ \\
$\begin{array}{l}\text { Problemas de } \\
\text { adaptaciónjambiente }\end{array}$ & $8.6 \%$ \\
$\begin{array}{l}\text { Dificultades por } \\
\text { distancia, tiempo }\end{array}$ & $4.3 \%$ \\
$\begin{array}{l}\text { duración de la } \\
\text { carrera }\end{array}$ & \\
$\begin{array}{l}\text { Situaciones } \\
\text { personales o }\end{array}$ & $2.6 \%$ \\
$\begin{array}{l}\text { familiares } \\
\text { oferta laboral } \\
\text { Inconformidad con } \\
\text { sede/U. (cupos, } \\
\text { horarios) } \\
\text { Total }\end{array}$ & $0.9 \%$ \\
\hline
\end{tabular}

Fuente: Elaboración propia.

Ante la pregunta abierta por qué ingresó a la carrera sin ser de su preferencia vocacional, se observa en la Tabla 5, que la principal razón fue ingresar a esta universidad estatal para tener la oportunidad de cambiar, posteriormente, a la de su preferencia. Igual, es importante razones más externas como el prestigio de la institución, cercanía geográfica y presión familiar. 
TABLA 5

Razón por la cual ingresó a la carrera a pesar de que no le despertaba interés. $(n=46)$

\begin{tabular}{lc}
\hline Razones & $\%$ \\
\hline $\begin{array}{l}\text { Para cambiarse } \\
\text { de carrera }\end{array}$ & \\
$\begin{array}{l}\text { posteriormente } \\
\text { Cercania entre }\end{array}$ & $23.9 \%$ \\
la U. y su & \\
hogar & $10.9 \%$ \\
$\begin{array}{l}\text { Desinformación } \\
\text { de lo que }\end{array}$ & \\
$\begin{array}{l}\text { Consistía la } \\
\text { carrera }\end{array}$ & $10.9 \%$ \\
$\begin{array}{l}\text { Inseguridad de } \\
\text { lo que deseaba }\end{array}$ & $10.9 \%$ \\
$\begin{array}{l}\text { Prestigio e } \\
\text { imagen de la }\end{array}$ & \\
$\begin{array}{l}\text { Carrera o la U. } \\
\text { Unica opción }\end{array}$ & $10.9 \%$ \\
$\begin{array}{l}\text { que tenía } \\
\text { Presión }\end{array}$ & $15.2 \%$ \\
familiar & $4.3 \%$ \\
Otra razón & $13.0 \%$ \\
Total & $100.0 \%$ \\
\hline
\end{tabular}

Fuente: Elaboración propia.

Con respecto a estas razones, se destacan las siguientes respuestas de algunos y algunas estudiantes:

E.451 (comunicación personal, 8 de setiembre, 2016): En realidad no era que la deseaba poco, si no que no se me brindó la información necesaria para saber de esta carrera, cuando la elegí fue porque era mi mejor opción en extraordinaria, el fin era cambiarme de carrera, pero al final me gustó mucho y decidí no cambiarme.

E.401 (comunicación personal, 8 de setiembre, 2016): Porque era la única que conocía en ese momento, no sabía nada acerca de las demás carreras.

E.51 ((comunicación personal, 6 de octubre, 2016): No sabía lo que quería estudiar.

Otras variables incluidas en este instrumento se relacionaban con algunas características sociodemográficas del estudiantado, específicamente, con la condición económica que se detalla en la Tabla 6. 
María Teresa Hernández-Jiménez, et al. Estudio descriptivo de Variables Sociodemográficas y motiva...

TABLA 6

Porcentajes de estudiantes desertores y no desertores en las cohortes 2011, 2012 y 2013 según variables sociodemográficas

\begin{tabular}{|c|c|c|c|}
\hline Ítems & $\begin{array}{l}\text { No } \\
\text { desertor }\end{array}$ & Desertor & $\mathrm{N}$ \\
\hline \multicolumn{4}{|l|}{$\begin{array}{l}\text { Ingresó a la U. } \\
\text { teniendo trabajo }\end{array}$} \\
\hline Si & $64,9 \%$ & $35,1 \%$ & 37 \\
\hline $\begin{array}{l}\text { No pero tuve luego } \\
\text { No y tampoco }\end{array}$ & & $28,0 \%$ & 50 \\
\hline $\begin{array}{l}\text { luego } \\
\text { Ingresó a la U. } \\
\text { teniendo hijos o } \\
\text { tuvo alguno } \\
\text { durante el tiempo } \\
\text { de estudio. }\end{array}$ & $76,4 \%$ & $23,6 \%$ & 462 \\
\hline No & $75,1 \%$ & $24,9 \%$ & 503 \\
\hline Si & $75,0 \%$ & $25,0 \%$ & 16 \\
\hline $\begin{array}{l}\text { Disfrutó de alguna } \\
\text { beca en el colegio } \\
\text { por condición } \\
\text { económica }\end{array}$ & & & \\
\hline No & $73,8 \%$ & $26,3 \%$ & 320 \\
\hline Sí & $77,4 \%$ & $22,6 \%$ & 199 \\
\hline \multicolumn{4}{|l|}{ Cuando ingresó a } \\
\hline \multicolumn{4}{|l|}{ la U. contaba con } \\
\hline \multirow{2}{*}{\multicolumn{4}{|c|}{$\begin{array}{l}\text { pudiera costear } \\
\text { todo el gasto de }\end{array}$}} \\
\hline & & & \\
\hline \multicolumn{4}{|l|}{ sus estudios para } \\
\hline \multicolumn{4}{|l|}{$\begin{array}{l}\text { solamente a } \\
\text { estudiar }\end{array}$} \\
\hline No & $74,4 \%$ & $25,6 \%$ & 207 \\
\hline Sí & $75,6 \%$ & $24,4 \%$ & 312 \\
\hline \multicolumn{4}{|l|}{ Cuando ingresó a } \\
\hline \multicolumn{4}{|l|}{ la U. contaba con } \\
\hline \multicolumn{4}{|l|}{$\begin{array}{l}\text { algún familiar o } \\
\text { miembro de su }\end{array}$} \\
\hline \multicolumn{4}{|l|}{ hogar que lo } \\
\hline apoyaba & & & \\
\hline \multicolumn{4}{|l|}{ moralmente e } \\
\hline \multicolumn{4}{|l|}{$\begin{array}{l}\text { incondicionalmente } \\
\text { para que }\end{array}$} \\
\hline \multicolumn{4}{|l|}{ estudiara la } \\
\hline \multicolumn{4}{|l|}{$\begin{array}{l}\text { Carrera a la cual } \\
\text { ingresó }\end{array}$} \\
\hline No & $72,4 \%$ & $27,6 \%$ & 76 \\
\hline Sí & $75,6 \%$ & $24,4 \%$ & 443 \\
\hline \multicolumn{4}{|l|}{ En la U. hay } \\
\hline \multicolumn{4}{|l|}{ estudiantes de } \\
\hline se les asigna un & & & \\
\hline estudiante & & & \\
\hline avanzado para & & & \\
\hline que les aconseje y & & & \\
\hline les comparta & & & \\
\hline experiencias. & & & \\
\hline Cuando usted & & & \\
\hline ingresó, recibió & & & \\
\hline algún tipo apoyo o & & & \\
\hline acompañamiento & & & \\
\hline por parte de un & & & \\
\hline $\begin{array}{l}\text { estudiante } \\
\text { avanzado. }\end{array}$ & & & \\
\hline No & $75,5 \%$ & $24,5 \%$ & 425 \\
\hline Sí & $73,4 \%$ & $26,6 \%$ & 94 \\
\hline
\end{tabular}


Fuente: Elaboración propia.

En general, las características relacionadas con la tenencia de hijos, la condición de becados en el colegio, el apoyo financiero o moral de otras personas y el acompañamiento de un estudiante avanzado cuando ingresó a esta universidad estatal fueron similares entre ambos grupos de estudiantes. La mayor diferencia fue la condición laboral, específicamente, el 35,1\% del estudiantado que sí tenía un trabajo al ingresar a la universidad abandonó sus estudios.

\section{TABLA 7}

Porcentajes de estudiantes desertores y no desertores de las cohortes 2011, 2012 y 2013 según nivel educativo de miembros del hogar

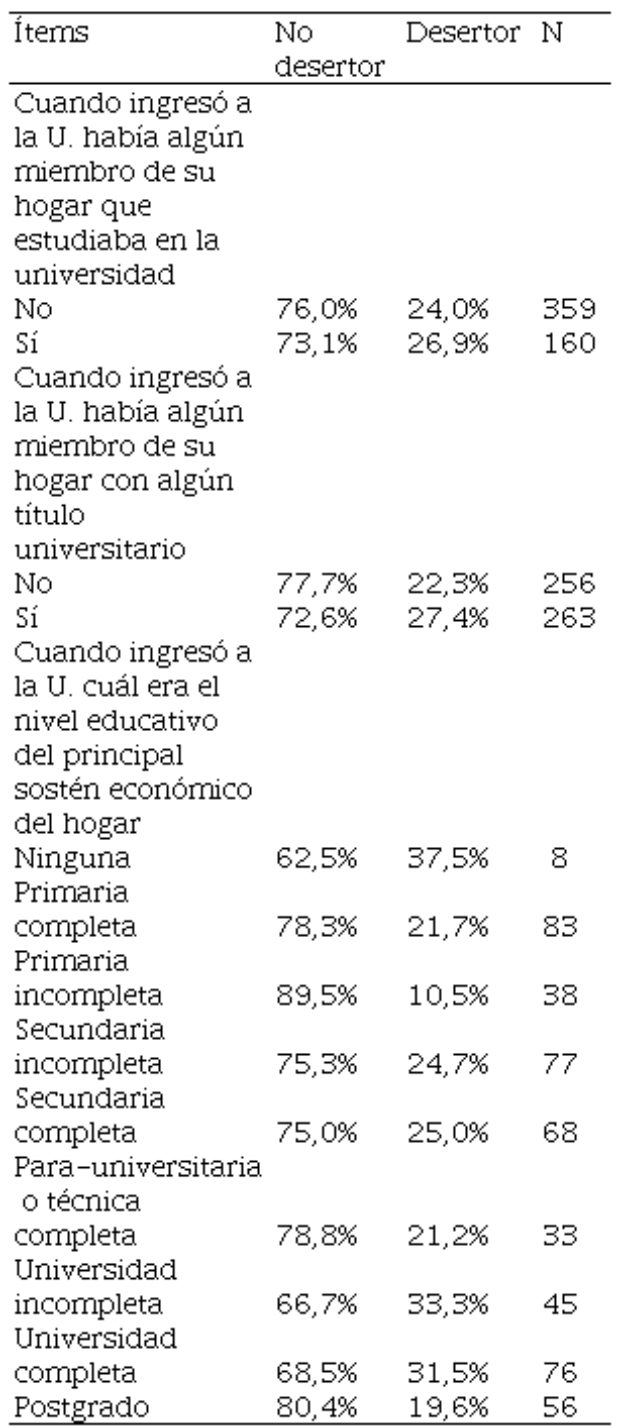

Fuente: Elaboración propia.

De acuerdo con los datos de las tres variables de la Tabla 7, quienes indicaron que el principal sostén familiar no tenía ningún nivel educativo, el 37,5\% abandonó sus estudios; en tanto que, aquellos con algún miembro del hogar titulado en la universidad desertaron un 27,4\% y la tendencia de deserción fue similar en aquellos hogares donde había un estudiante universitario. En estas tres variables no se encontraron evidencias con la prueba de la chi-cuadrada. 
María Teresa Hernández-Jiménez, et al. Estudio descriptivo de Variables Sociodemográficas y motiva...

TABLA 8

Razones por las que desertaron o pensaron desertar de la universidad (U).

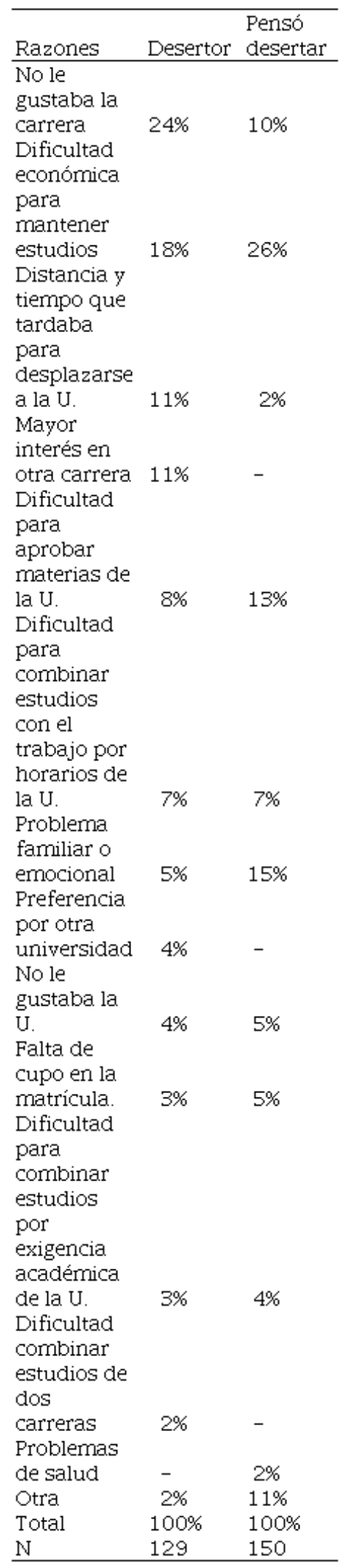


Fuente: Elaboración propia.

En esta Tabla 8 se sistematiza las respuestas abiertas de los participantes acerca de principal razón para abandonar sus estudios en esta universidad estatal $(\mathrm{U})$, tanto en la muestra de desertores como en quienes lo pensaron en algún momento (38,5\%). La insatisfacción con la carrera y la condición socioeconómica, especialmente, en quienes tenían la intención de abandonar los estudios fueron las principales razones. La tercera fue el tiempo de traslado a la institución, por ejemplo, las y los estudiantes desertores, en promedio, tardaron 62 minutos, en tanto que los no desertores, tardaban en promedio 54 minutos para para llegar a la universidad cuando ingresó.

\section{Conclusiones}

En relación con las variables motivacionales se encontró que la condición de abandono de los estudios se asoció con el poco interés por estudiar en la carrera en la cual fue admitido, la matrícula a una carrera no deseada y que no era parte de la oferta académica de esta universidad, el deseo de estudiar en otra institución de educación superior y por la disminución de estudiar la carrera y en esta universidad estatal cuando comenzó a llevar los cursos. Estos hallazgos coinciden con los estudios de Canales y De los Ríos (2007), Aranzazu y Rojas (2018); Casanova et al. (2018), quienes apuntan que al tomar la decisión de ingresar a la educación superior las personas jóvenes desconocen o tienen poca información sobre el significado de estudiar una carrera universitaria. Además, destacan que las consecuencias de estudiar una carrera no satisfactoria de sus expectativas influye negativamente en su motivación académica. En este sentido Casanova et al. (2018) también concluyen que el ajuste vocacional es la principal causa de abandono para los grupos según diferentes años de ingreso.

Además, se logró profundizar que la pérdida de interés por continuar sus estudios fue que no le gustó la carrera y no llenaba sus expectativas (46,6\%). Esta falta de claridad vocacional al ingresar a la universidad resultó ser una de las variables más importantes. Tal situación podría prevenirse desde una orientación vocacional en la educación secundaria, la cual le permita al alumnado tener más conocimiento de sus propias habilidades y perfil vocacional antes de seleccionar la carrera. Además, al igual que en otras universidades, hay estudiantes que se matriculan en una carrera sin ser de su preferencia vocacional, solo para lograr ingresar a la institución y luego tener la oportunidad de cambiarse internamente a otra carrera. Esta es otra variable relevante asociada a la deserción, puesto que no resulta fácil el cambio interno de carrera y sus opciones son más limitadas.

De las variables sociodemográficas, ninguna variable mostró una asociación con el abandono. No obstante, $35 \%$ del estudiantado que ingresó a esta universidad estatal teniendo un trabajo y $28 \%$ de aquellos que ingresaron y luego obtuvieron un trabajo desertaron; en contraste con un $76 \%$ de estudiantes activos que se dedicaron tiempo completo a sus estudios universitarios. Como lo destacan Vries, León, Romero y Hernández (2011) la deserción se debe en gran medida a la incompatibilidad de estudios y trabajo, y a la falta de vocación. En estudios similares, (Giovagnoli, 2002; Lopera 2008; Regueyra 2013; Casanova et al. 2018) comprobaron que haber iniciado los estudios universitarios y simultáneamente estar trabajando provoca un mayor riesgo de abandono en relación con quienes inician sus estudios sin una responsabilidad laboral.

En este estudio, la variable condición económica no se asoció con el abandono, no obstante, los desertores indicaron que, entre otras razones, tenían dificultades económicas para continuar sus estudios. Al respecto, González (2006) señala que las condiciones socioeconómicas es una de las principales razones de la deserción (el lugar de residencia, nivel de ingresos, nivel educativo de los padres, el ambiente familiar, la necesidad de trabajar para mantenerse o aportar a su familia), especialmente, en los quintiles de menores ingresos.

Las otras variables tales como la tenencia de hijos, tiempo de traslado, lugar de residencia, el apoyo familiar y acompañamiento en la universidad, no mostraron evidencias estadísticas en esta muestra de estudiantes; 
sin embargo, en múltiples estudios se ha encontrado una relación con el tiempo de traslado (Kuna, García y Villatoro, 2010). Las variables relacionadas con el nivel educativo de los miembros familiares tampoco mostraron evidencias estadísticas; sin embargo, es interesante que, en promedio, el $77 \%$ de estudiantes activos no tenían miembros de su hogar estudiando en la universidad, ni un título universitario. En estudios similares se ha encontrado que cuanto menor nivel educativo de los padres mayor riesgo de deserción (Giovagnoli, 2002).

En suma, los resultados de este estudio se deben interpretar en el contexto de estudiantes que abandonaron sus estudios universitarios o que en algún momento pensaron desertar, pero continuaron. Como bien lo apuntan, Villamizar y Pérez (2011) y Casanova, Díaz, Soto, García y Miranda (2018), en los casos de desertores del sistema universitario intervienen variables personales, familiares y económicas, pero en aquellos desertores de la universidad y de la carrera, la orientación vocacional influyó de cierta forma.

En cuanto a la relevancia de estos hallazgos, cabe destacar que a partir de la presentación de los resultados de esta investigación ante diversas instancias y autoridades de la universidad se tomaron decisiones para atender esta problemática. A modo de ejemplo, el Consejo Institucional (máxima instancia de toma de decisiones de la universidad), aprobó el Plan Anual Operativo Institucional para el año 2019 con dos nuevas metas para articular las acciones institucionales de todas las dependencias docentes. La primera de ellas dicta: "Disminuir en 2 puntos porcentuales la reprobación anualizada de los cursos de grado con respecto al año anterior" (Oficina de Planificación Institucional, 2018, 67-68) y la segunda establece: "Disminuir en 2 puntos porcentuales la tasa de deserción de primer ingreso en el I semestre, al mismo periodo del año anterior" (Oficina de Planificación Institucional, 2018, 67-68). A partir de la primera directriz, cada una de las veintitrés escuelas formularon acciones concretas para su cumplimiento; por ejemplo, en el primer semestre del año 2019, se han implementado acciones tales como:

- Identificación de los cursos con mayor reprobación.

- Servicio de tutorías para los cursos con mayor dificultad con el requisito de asistencia obligatoria por parte del estudiantado.

- Asistencia a talleres de apoyo para el desarrollo de proyectos.

- Actualización de los programas de los cursos sobre objetivos, atributos, contenidos, evaluación y material didáctico, desde la perspectiva de las habilidades blandas.

- Revisión y seguimiento periódico del rendimiento académico individual.

- Capacitación al personal docente en estrategias didácticas.

En cuanto a las acciones efectuadas en función de la segunda meta, sobre la disminución de la deserción, varias dependencias docentes han desarrollado lo siguiente.

- Coordinación de acciones y capacitación a estudiantes del programa de mentoría para que apoyen con información y guía a la población de primer ingreso para evitar su deserción.

- Realización de talleres de apoyo a la población de primer ingreso.

- Seguimiento del indicador de deserción mediante el Sistema de Indicadores de Gestión (SIGI).

- Organización de charlas motivacionales por parte de egresados y expertos en diversas áreas, dirigidas a estudiantes regulares.

- Organización de actividades de integración y recreación, incluyendo un convivio con los egresados, estudiantes regulares y profesores.

- Analizar la condición a estudiantes que han tramitado retiros justificados, retiros especiales, abandono de cursos, reprobación por ausencias y cambios de carrera.

- Charlas a estudiantes de primer ingreso por parte de estudiantes avanzados en la carrera, abordando temas de: giras de campo, vida estudiantil, actividades extracurriculares, importancia de la asesoría curricular, entre otros.

- Seguimiento a estudiantes de bajo rendimiento académico, que llevan tutorías y talleres. 
El resultado final del cumplimiento de ambas metas se obtendrá en el mes de febrero del año 2020; sin embargo, al efectuar un monitoreo trimestral e indicar el porcentaje de avance a las autoridades institucionales, se puede lograr su efectivo cumplimiento.

\section{REFERENCIAS}

Aguiar, E., Ambrose, G.A., Chawla, N.V., Goodrich, V. y Brockman, J. (2014). Engagement vs Performance: Using Electronic Portfolios to Predict First Semester Engineering Student Persistence. Journal of Learning Analytics, 1(3), 7-33. Recuperado de https://files.eric.ed.gov/fulltext/EJ1126984.pdf

Aranzazu, D.G. y Rojas, M.D. (2018). Aproximación a la Deserción Femenina del Programa Ingeniería Administrativa en La Facultad de Minas de la Universidad Nacional de Colombia 2010-2017. Conferencia Latinoamericana sobre Abandono en la Educación Superior. Ponencia del Congreso CLABES VIII, Ciudad Panamá. Recuperado de https://revistas.utp.ac.pa/index.php/clabes/article/view/1926

Barquero, J.A. (1993). Deserción universitaria, revisión bibliográfica. Cartago, Costa Rica: Instituto Tecnológico de Costa Rica. Recuperado de http://hdl.handle.net/2238/690

Barquero, J.A. (1997). Rendimiento académico, deserción y graduación en el ITCR. Cartago, Costa Rica: Instituto Tecnológico de Costa Rica. Recuperado de http://hdl.handle.net/2238/701

Brenes, M. I. (2005). Deserción y repitencia en la educación superior universitaria de Costa Rica. San José, Costa Rica: Consejo Nacional de Rectores.

Canales, A. y De los Ríos, D. (2007). Factores explicativos de la deserción universitaria. Calidad en la Educación, (26), 173-201. doi:https://doi.org/10.31619/caledu.n26.239

Casanova, D., García, D. y Miranda, C. (2018). Motivos de abandono de los estudiantes de la Universidad Católica de la Santísima Concepción. Conferencia Latinoamericana sobre Abandono en la Educación Superior. Ponencia del Congreso CLABES VIII, Ciudad Panamá. Recuperado de https://revistas.utp.ac.pa/index.php/clabes/arti cle/view/1911

Casanova, M.P., Díaz, A., Soto, P. García, D. y Miranda, C. (2018). Deserción no asumida: un fenómeno Psicosocial difícil de pesquisa. Conferencia Latinoamericana sobre Abandono en la Educación Superior. Ponencia del Congreso CLABES VIII, Ciudad Panamá. Recuperado de https://revistas.utp.ac.pa/index.php/clabes/article/ view/1893

Consejo Nacional de Rectores [CONARE] (2008). Memoria de la Cátedra UNESCO de Dirección Universitaria. Indicadores del Sistema Universitario Público de Costa Rica en el ámbito académico. San José, Costa Rica: CONARE

Delen, D. (2010). A comparative analysis of machine learning techniques for student retention management. Decision Support Systems, 49(4), 498-506. Recuperado de https://www.researchgate.net/publication/223457886

Dirección de Servicios Estudiantiles y Académicos [DISEA] (1980a). Estudio deserción correspondiente al I Semestre 1978. Cartago, Costa Rica: Instituto Tecnológico de Costa Rica.

Dirección de Servicios Estudiantiles y Académicos [DISEA] (1980b). Estudio deserción correspondiente al año 1979. Cartago, Costa Rica: Instituto Tecnológico de Costa Rica.

Dirección de Servicios Estudiantiles y Académicos [DISEA] (1981). Estudio deserción correspondiente al curso lectivo 1980. Cartago, Costa Rica: Instituto Tecnológico de Costa Rica.

Giovagnoli, P.I. (2002). Determinantes de la deserción y graduación universitaria: Una aplicación utilizando modelos de duración (Tesis de maestría). Universidad Nacional de La Plata, Argentina. Recuperada de http://sedici.unlp.e du.ar/handle/10915/3436

González, J. (2006). Repetición y deserción universitaria en América Latina. Recuperado de https://bit.ly/2WeNWRF

Jia, J.W. (2013). Machine learning algorithms and predictive models for undergraduate student retention. En Proceedings of the World Congress on Engineering and Computer Science. International Association of Engineers, San Francisco, USA. Recuperado de https://bit.ly/2Nu6YMj 
Kuna, H., García, R. y Villatoro, F.R. (2010). Identificación de Causales de Abandono de Estudios Universitarios. Uso de Procesos de Explotación de Información. Revista Iberoamericana de Tecnología en Educación y Educación en Tecnologia, (5), 39-44. Recuperado de https://bit.ly/2VOFWTG

Lopera, C. (2008). Determinantes de la deserción universitaria en la Facultad de Economía de la Universidad del Rosario. Serie Documentos. Borradores de Investigación, (95), 1-40. Recuperado de https://bit.ly/2P2Q3TP

Mesarić, J. y Šebalj, D. (2016). Decision trees for predicting the academic success of Students. Croatian Operational Research Review, 7(2), 367-388. doi; https://doi.org/10.17535/crorr.2016.0025

Miranda, M.A. y Guzmán, J. (2017). Análisis de la Deserción de Estudiantes Universitarios usando Técnicas de Minería de Datos. Formación Universitaria, 10(3), 61-68. doi: http://dx.doi.org/10.4067/S0718-5006201700 0300007

Nakhkob, B. y Khademi, M. (2016). Predicted Increase Enrollment in Higher Education Using Neural Networks and Data Mining Techniques. Journal of Advances in Computer Research, 7(4), 125-140. Recuperado de https://b it.ly/2I8A7tR

Oficina de Planificación Institucional (2018). Plan anual operativo 2019. Instituto Tecnológico de Costa Rica. Recuperado de https://www.tec.ac.cr/sites/default/files/media/doc/plan_anual_operativo_2019_0.pdf

Oñate, A. (2016). Análisis de la deserción y permanencia académica en la educación superior aplicando mineria de datos (Tesis de Maestría) Universidad Nacional de Colombia-Sede Bogotá. Recuperado de http://bdigital.unal.edu.c o/53635/1/alvaroagustino\%C3\%B1atebowen.2016.pdf

Oztekin, A. (2016). A hybrid data analytic approach to predict college graduation status and its determinative factors. Industrial Management \& Data Systems, 116(8), 1678-1699. doi: https://doi.org/10.1108/IMDS-09-2015-03 63

Plagge, M. (2013). Using artificial neural networks to predict first-year traditional students second year retention rates. En Proceedings of the 51st ACM Southeast Conference ACM, Ponencia llevada a cabo en la Armstrong Atlantic State University, Georgia, USA. doi: https://doi.org/10.1145/2498328.2500061

Regueyra, M.G. (2013). Aprendizaje sobre la población estudiantil que no continúa y el derecho a la Educación Superior. Conferencia Latinoamericana sobre Abandono en la Educación Superior. Ponencia del Congreso CLABES III, Ciudad Panamá. Recuperado de https://bit.ly/2BYEZ2c

Regueyra, G. y Rojas, C. (2013). Una mirada a la permanencia de la población estudiantil de trabajo social. Revista Actualidades Investigativas en Educación, 13(3), 1-25. Recuperado de https://bit.ly/32WOiLT

Rodríguez, M. y Zamora J.A. (2014). Análisis de la deserción en la Universidad Nacional desde una perspectiva longitudinal. Quinto Informe Estado de la Educación 2015. Recuperado de http://repositorio.conare.ac.cr/han dle/20.500.12337/829

Tinto, V. (1989). Definir la deserción: una cuestión de perspectiva. Revista de Educación Superior, 71, 1.9. Recuperado de http://publicaciones.anuies.mx/pdfs/revista/Revista71_S1A3ES.pdf

Torres, I.S. (2018). Estudio Longitudinal Permanencia y Abandono en Universitarios (2015-2019). Corporación Universitaria Autónoma del Cauca-Colombia. Conferencia Latinoamericana sobre Abandono en la Educación Superior. Ponencia del Congreso CLABES VIII, Ciudad Panamá. Recuperado de https://revistas.utp.ac.pa/ind ex.php/clabes/article/view/2050

Treviño, M., Ibarra, S., Castán, J., Laria, J. y Guzmán, J. (2013). A framework to avoid scholar desertion using artificial intelligence. En World Congress on Engineering III, International Association of Engineers, London, UK. Recuperado de http://www.iaeng.org/publication/WCE2013/WCE2013_pp1493-1497.pdf

Villamizar, G.A. y Pérez, L. (2011). Identificación de factores motivacionales y sociodemográficos de estudiantes desertores de la Facultad de Psicología de la Universidad Pontificia Bolivariana Bucaramanga. Psicogente, 14(25). Recuperado de http://revistas.unisimon.edu.co/index.php/psicogente/article/view/1862

Vries, W., León, P., Romero, J.F. y Hernández, I. (2011). ¿Desertores o decepcionados? Distintas causas para abandonar los estudios universitarios. Revista Educación Superior, 40(160), 29-49. Recuperado de https://bit.l $\mathrm{y} / 1 \mathrm{TzOzru}$ 
Revista Educación, 2020, 44(1), Enero-Junio, ISSN: 0379-7082 / 2215-2644

CC BY-NC-ND 